\title{
Outage Minimization In Cognitive Radio Networks With Limited Feedback
}

\author{
YuanYuan He and Subhrakanti Dey, Senior Member, IEEE \\ Department of Electrical and Electronic Engineering \\ University of Melbourne, Vic. 3010, Australia \\ e-mail: $\{$ yyhe, sdey\}@ee.unimelb.edu.au
}

\begin{abstract}
We address an optimal transmit power allocation problem that minimizes the outage probability of a secondary user (SU) who is allowed to coexist with a primary user (PU) in a narrowband spectrum sharing cognitive radio network, under a long term average transmit power constraint at the secondary transmitter (SU-TX) and an average interference power constraint at the primary receiver (PU-RX), with quantized channel state information (CSI) (including both the channels from SU-TX to SU-RX, denoted as $g_{1}$ and the channel from SU-TX to PU-RX, denoted as $g_{0}$ ) at the SU-TX. The optimal quantization regions in the vector channel space is shown to have a 'stepwise' structure. With this structure, the above outage minimization problem can be explicitly formulated and solved by employing the Karush-Kuhn-Tucker (KKT) necessary optimality conditions to obtain a locally optimal quantized power codebook. A low-complexity near-optimal quantized power allocation algorithm is derived for the case of large number of feedback bits. More interestingly, we show that as the number of partition regions approaches infinity, the length of interval between any two adjacent quantization thresholds on the $g_{0}$ axis is asymptotically equal when the average interference power constraint is active. Similarly, we show that when the average interference power constraint is inactive, the ratio between any two adjacent quantization thresholds on the $g_{1}$ axis becomes asymptotically identical. Finally, an explicit expression for the asymptotic SU outage probability at high rate quantization (as the number of feedback bits goes to infinity) is also provided, and is shown to approximate the optimal outage behavior extremely well for large number of bits of feedback via numerical simulations. Numerical results also illustrate that with 6 bits of feedback, the derived algorithms provide SU outage performance very close to that with full CSI at SU-TX.
\end{abstract}

\section{INTRODUCTION}

Scarcity of available vacant spectrum is limiting the growth of wireless products and services [1]. Traditional spectrum licensing policy forbids unlicensed users to transmit in order to avoid unfavorable interference at the cost of spectral utilization efficiency. This led to the idea of cognitive radio (CR) technology, originally introduced by J. Mitola [2], which holds tremendous promise to dramatically improve the efficiency of spectral utilization.

The key idea behind $\mathrm{CR}$ is that an unlicensed/secondary user (SU) is allowed to communicate over a frequency band originally licensed to a primary user (PU), as long as the transmission of SU does not generate unfavorable impact on the operation of PU in that band. Effectively, three categories of CR network paradigms have been proposed: interweave, overlay, and underlay [3]. In the underlay systems, also known as spectrum sharing model, which is the focus of this paper, the SU can transmit even when the PU is present, but the transmitted power of SU should be controlled properly so as to ensure that the resulting interference does not degrade the received signal quality of PU to an undesirable level [4] by imposing the so called interference temperature [5] constraints at PU (average or peak interference power (AIP/PIP) constraint) and as well as to enhance the performance of SU transmitter (SU-TX) to SU receiver (SU-RX) link.

Various notions of capacity for wireless channels include ergodic capacity (for delay-insensitive services), delaylimited capacity and outage probability (for real-time applications). These information theoretic capacity notions constitute important performance measures in analyzing the performance limits of CR systems. In [5], the authors investigated the ergodic capacity of such a dynamic narrowband spectrum sharing model under either AIP or PIP constraint at PU receiver (PU-RX) in various fading environments. In [6], the authors studied optimum power allocation for three different capacity notions under both AIP and PIP constraints. In [4], the authors also considered the average or peak transmit power (ATP/PTP) constraint at SU-TX and investigated the optimal power allocation strategies to achieve the ergodic capacity and outage capacity of SU under various combinations of secondary ATP/PTP constraints and AIP/PIP constraints.

Achieving the optimal system performance requires the SU-TX to acquire full channel state information (CSI) including the channel information from SU-TX to PU-RX and the channel information from SU-TX to SU-RX. Most of the above results assume perfect knowledge or full CSI, which is very difficult to implement in practice, especially the channel information from SU-TX to PU-RX without PU's cooperation. A few recent papers have emerged that address this concern by investigating performance analysis with various forms of partial CSI at SU-TX, such as noisy CSI and quantized CSI. With assumption of perfect knowledge of the CSI from SU-TX to SU-RX channel, [7] and [8] studied the effect of imperfect CSI (in the form of noisy channel estimate) of the SU-TX to PU-RX channel under AIP or PIP constraint. Recently, [9] has proposed a practical design paradigm for cognitive beamforming based on finite-rate cooperative feedback from the PU-RX to the SU-TX and cooperative feedforward from the SU-TX to the PU-RX. A robust cognitive beamforming scheme was also analyzed in [10], where full channel information on SU-TX to SU-RX channel was assumed, and the imperfect channel information on the SU-TX to PU-RX channel was modelled using an uncertainty set. [11] studied the issue of channel quantization for resource allocation via the framework of utility maximization in OFDMA based CR networks, but did not investigate the joint channel partitioning and rate/power codebook design problem. In our earlier work [12], we addressed a SU ergodic capacity maximization problem in a wideband spectrum sharing scenario with quantized infor- 
mation about the vector channel space involving the SU-TX to SU-RX channel and the SU-TX to PU-RX channel over all bands, under an ATP constraint at SU-TX and an AIP at the PU-RX.

In this paper, we address the problem of minimizing the SU outage probability under an ATP constraint at the SUTX and an AIP constraint at the PU-RX with only quantized CSI available at SU-TX. We consider an infrastructurebased narrowband spectrum sharing scenario where a SU communicates to its base station (SU-BS) on a narrowband channel shared with a PU communicating to its receiver PURX contained within the primary base station (PU-BS). As in [12], a central controller called the CR network manager is assumed to exist, so that the optimal partition regions of the vector channel space (consisting of the SU-TX to SURX channel (denoted by power gain $g_{1}$ ) and the interfering channel between the SU-TX and PU-RX (denoted by power gain $\left.g_{0}\right)$ ) and the corresponding optimal power codebook can be jointly designed, based on the channel statistics. Under these networking assumptions, we prove a 'stepwise' structure of the optimal channel partition regions, which helps us explicitly formulate the outage minimization problem and solve it using the corresponding Karush-Kuhn-Tucker (KKT) necessary optimality conditions. As the number of feedback bits go to infinity, we show that the power level for the last region approaches zero, allowing us to derive a useful lowcomplexity suboptimal quantized power allocation algorithm called 'ZPiORA' for high rate quantization. We also derive some other useful properties related to the channel quantizer structure as the number of feedback bits approaches infinity: (a) under an active AIP constraint, the length of interval between any two adjacent quantization thresholds on $g_{0}$ axis is asymptotically the same, and (b) while when the AIP is inactive, the ratio between any two adjacent quantization thresholds on $g_{1}$ axis asymptotically becomes identical. Finally, with these properties, we derive explicit expressions for asymptotic (as the number of feedback bits increase) behavior of the SU outage probability with quantized power allocation for large resolution quantization.

This paper is organized as follows. Section II introduces the system model and the problem formulation based on the full CSI assumption. Section III presents the joint design of the optimal channel partition regions and an optimal power codebook algorithm. A low-complexity suboptimal quantized power allocation strategy is also derived using novel interesting properties of the quantizer structure and optimal quantized power codebooks. In Section IV, the asymptotic behavior of SU outage probability for high resolution quantization is investigated. Simulation results are given in Section $\mathrm{V}$, followed by concluding remarks in Section VI. Proofs of various results are excluded due to space limitations, but can be found in a longer version of this paper [16].

\section{System Model AND PROBlem Formulation}

We consider an infrastructure-based spectrum sharing network where a SU communication uplink to the SU-BS coexists with a PU link (to the PU-BS) within a narrowband channel. Regardless of the on/off status of PU, the SU is allowed to access the band which is originally allocated to PU, so long as the impact of the transmission of SU does not reduce the received signal quality of PU below a prescribed level. All channels here are assumed to be Rayleigh block fading channels. Let $g_{1}$ and $g_{0}$ denote the nonnegative realvalued instantaneous channel power gains for the links from SU-TX to SU-RX and SU-TX to PU-RX respectively. $g_{1}$ and $g_{0}$ are statistically mutually independent and, without loss of generality (w.l.o.g), are assumed to have unity mean. The additive noises for each channel are independent Gaussian random variables with, w.l.o.g, zero mean and unit variance. For analytical simplicity, the interference from the primary transmitter (PU-TX) to SU-RX is neglected following previous work such as [4], [5].

Given a channel realization $\left(g_{0}, g_{1}\right)$, let the instantaneous transmit power (with full CSI) at the SU-TX be represented by $p\left(g_{0}, g_{1}\right)$, then the maximum mutual information of the SU for this narrowband spectrum sharing system can be expressed as $R\left(g_{1}, p\left(g_{0}, g_{1}\right)\right)=\frac{1}{2} \log \left(1+g_{1} p\left(g_{0}, g_{1}\right)\right)$, where log represents the natural logarithm. The outage probability of SU-TX with a pre-specified transmission rate $r_{0}$, is given as, $P_{\text {out }}=\operatorname{Pr}\left\{R\left(g_{1}, p\left(g_{0}, g_{1}\right)\right)<r_{0}\right\}$, where $\operatorname{Pr}\{A\}$ indicates the probability of event $A$ occurring. Using the interference temperature concept in [5], a common way to protect PU's received signal quality is by imposing either an average or a peak interference power (AIP/PIP) constraint at the PU-RX. In [13], it was demonstrated that the AIP constraint is more flexible and favorable than the PIP constraint in the context of transmission over fading channels. Let $Q_{a v}$ denote the average interference power limit tolerated by PU-RX, then the AIP constraint can be written as, $E\left[g_{0} p\left(g_{0}, g_{1}\right)\right] \leq Q_{a v}$.

The following optimal power allocation problem that minimizes the outage probability of SU in a narrowband spectrum sharing with one PU, under both a long term average transmit power (ATP) constraint at SU-TX and an AIP constraint at the PU-RX, was considered in [4]

$$
\begin{array}{cl}
\min _{p\left(g_{0}, g_{1}\right) \geq 0} & \operatorname{Pr}\left\{\frac{1}{2} \log \left(1+g_{1} p\left(g_{0}, g_{1}\right)\right)<r_{0}\right\} \\
\text { s.t. } & E\left[p\left(g_{0}, g_{1}\right)\right] \leq P_{a v}, \\
& E\left[g_{0} p\left(g_{0}, g_{1}\right)\right] \leq Q_{a v}
\end{array}
$$

where $P_{a v}$ is maximum average transmit power at SU-TX. With the assumption that perfect CSI of both $g_{0}$ and $g_{1}$ is available at the SU-TX, the optimal power allocation scheme for Problem (1) is given by [4]

$$
p^{*}\left(g_{0}, g_{1}\right)=\left\{\begin{array}{lc}
\frac{c}{g_{1}}, & \lambda_{f}^{*}+\mu_{f}^{*} g_{0}<\frac{g_{1}}{c} \\
0, & \text { otherwise }
\end{array}\right.
$$

where $c=e^{2 r_{0}}-1$, and $\lambda_{f}^{*}, \mu_{f}^{*}$ are the optimal nonnegative Lagrange multipliers associated with the ATP constraint and the AIP constraint, respectively, obtained by solving $\lambda_{f}^{*}\left(E\left[p\left(g_{0}, g_{1}\right)\right]-P_{a v}\right)=0, \mu_{f}^{*}\left(E\left[g_{0} p\left(g_{0}, g_{1}\right)\right]-Q_{a v}\right)=0$.

However, the assumption of full CSI at the SU-TX (especially that of $g_{0}$ ) is usually unrealistic and difficult to implement in practical systems, especially when this channel is not time-division duplex (TDD). In the next section, we are therefore interested in designing a power allocation strategy of the outage probability minimization Problem (1) based on quantized CSI at the SU-TX acquired via a no-delay and error-free feedback link with limited rate. 


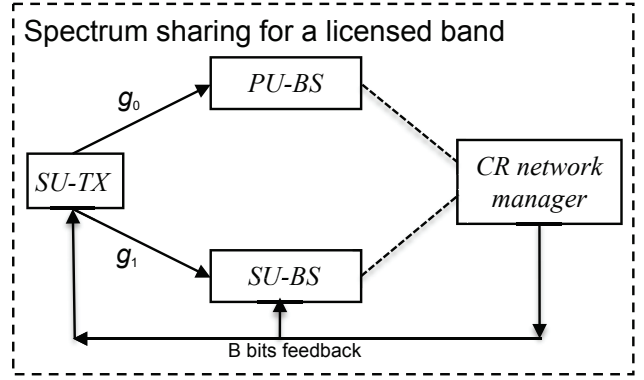

Fig. 1. System model for narrowband spectrum sharing scenario with limited rate feedback

\section{OPTIMUM QUANTIZED POWER ALLOCATION (QPA) WITH IMPERFECT $g_{1}$ AND $g_{0}$ AT SU-TX}

\section{A. Optimal QPA with limited rate feedback strategy}

As shown in Fig.1, following our earlier work [12], we assume that there is a central controller termed as CR network manager who can obtain perfect information of $g_{0}$ and $g_{1}$, from PU-RX at the PU base station and SU-RX at the SU base station respectively, possibly over fibre-optic links, and then forward some appropriately quantized $\left(g_{0}, g_{1}\right)$ information to SU-TX through a finite-rate feedback link. For further details on the justification of resulting benefits due this assumption, see [12]. Under such a network modelling assumption, given $\mathrm{B}$ bits of feedback, a power codebook $\mathcal{P}=\left\{p_{1}, \ldots, p_{L}\right\}$ of cardinality $L=2^{B}$, is designed offline purely on the basis of the statistics of $g_{0}$ and $g_{1}$ information at the CR network manager. This codebook is also known a priori by both SU-TX and SU-RX for decoding purposes. Given a channel realization $\left(g_{0}, g_{1}\right)$, the CR network manager employs a deterministic mapping from the current instantaneous $\left(g_{0}, g_{1}\right)$ information to one of $L$ integer indices (let $\mathcal{I}\left(g_{0}, g_{1}\right)$ denote the mapping, which partitions the vector space of $\left(g_{0}, g_{1}\right)$ into $L$ regions $\mathcal{R}_{1}, \ldots, \mathcal{R}_{L}$, defined as $\mathcal{I}\left(g_{0}, g_{1}\right)=j, \quad$ if $\left.\left(g_{0}, g_{1}\right) \in \mathcal{R}_{j}, \quad j=1, \ldots, L\right)$, and then sends the corresponding index $j=\mathcal{I}\left(g_{0}, g_{1}\right)$ to the SU-TX (and the SU-RX) via the feedback link. The SU-TX then uses the associated power codebook element (e.g., if the feedback signal is $j$, then $p_{j}$ will be used as the transmission power) to adapt its transmission strategy.

Define an indicator function $X_{j}, j=1, \ldots, L$, as

$$
X_{j}=\left\{\begin{array}{lc}
1, & \frac{1}{2} \log \left(1+g_{1} p_{j}\right)<r_{0} \\
0, & \text { otherwise. }
\end{array}\right.
$$

Let $\operatorname{Pr}\left(\mathcal{R}_{j}\right), E\left[\bullet \mid \mathcal{R}_{j}\right]$ represent $\operatorname{Pr}\left(\left(g_{0}, g_{1}\right) \in \mathcal{R}_{j}\right)$ and $E\left[\bullet \mid\left(g_{0}, g_{1}\right) \in \mathcal{R}_{j}\right]$, respectively. Then the SU outage probability minimization problem with limited feedback can be formulated as

$$
\begin{array}{ll}
\min _{p_{j} \geq 0, \mathcal{R}_{j} \forall j} & \sum_{j=1}^{L} E\left[X_{j} \mid \mathcal{R}_{j}\right] \operatorname{Pr}\left(\mathcal{R}_{j}\right) \\
\text { s.t. } & \sum_{j=1}^{L} E\left[p_{j} \mid \mathcal{R}_{j}\right] \operatorname{Pr}\left(\mathcal{R}_{j}\right) \leq P_{a v}, \\
& \sum_{j=1}^{L} E\left[g_{0} p_{j} \mid \mathcal{R}_{j}\right] \operatorname{Pr}\left(\mathcal{R}_{j}\right) \leq Q_{a v} .
\end{array}
$$

Thus the key problem to solve here is the joint optimization of the channel partition regions and the power codebook such that the outage probability of SU is minimized under the above constraints.

The dual problem of (4) is expressed as, $\max _{\lambda \geq 0, \mu \geq 0} g(\lambda, \mu)-\lambda P_{a v}-\mu Q_{a v}$, where $\lambda, \mu$ are the nonnegative Lagrange multipliers associated with the ATP and AIP constraints in Problem (4), and the Lagrange dual function $g(\lambda, \mu)$ is defined as

$$
\min _{p_{j} \geq 0, \mathcal{R}_{j}, \forall j} \sum_{j=1}^{L} E\left[X_{j}+\left(\lambda+\mu g_{0}\right) p_{j} \mid \mathcal{R}_{j}\right] \operatorname{Pr}\left(\mathcal{R}_{j}\right)
$$

The procedure we use to solve above dual problem is: Step 1: With fixed values of $\lambda$ and $\mu$, find the optimal solution (power codebook and quantization regions) for the Lagrange dual function (5).

Step 2: Find the optimal $\lambda$ and $\mu$ by solving the dual problem using subgradient search method, i.e, updating $\lambda, \mu$ until convergence using $\lambda^{l+1}=\left[\lambda^{l}-\alpha^{l}\left(P_{a v}-\right.\right.$ $\left.\left.\sum_{j=1}^{L} E\left[p_{j} \mid \mathcal{R}_{j}\right] \operatorname{Pr}\left(\mathcal{R}_{j}\right)\right)\right]^{+}, \quad \mu^{l+1}=\left[\mu^{l}-\beta^{l}\left(Q_{a v}-\right.\right.$ $\left.\left.\sum_{j=1}^{L} E\left[g_{0} p_{j} \mid \mathcal{R}_{j}\right] \operatorname{Pr}\left(\mathcal{R}_{j}\right)\right)\right]^{+}$, where $l$ is the iteration number, $\alpha^{l}, \beta^{l}$ are positive scalar step sizes for the $l$-th iteration satisfying $\sum_{l=1}^{\infty} \alpha_{l}=\infty, \sum_{l=1}^{\infty}\left(\alpha_{l}\right)^{2}<\infty$ and similarly for $\beta_{l}$, and $[x]^{+}=\max (x, 0)$.

A general method to solve Step 1 is to employ a simulation-based optimization algorithm called Simultaneous Perturbation Stochastic Approximation (SPSA) algorithm [14], where one can use the objective function of Problem (5) as the loss function and the optimal power codebook elements for each channel partition are obtained via a randomized stochastic gradient search technique. However due to the high computational complexity of SPSA and its long convergence time to solve Problem (5), we will next derive a lowcomplexity approach for solving Problem (5). Since the original problem (4) is not convex with respect to the power codebook elements, the solution we obtain is also locally optimal.

Remark 1: Note that due to the presence of the indicator function (non-differentiable) and no explicit expression being available for the outage probability with quantized power allocation, we cannot directly exploit the Generalized Lloyd Algorithm (GLA) with a Lagrangian distortion, as we used in [12], to solve Problem (5). Although we can use GLA with a sigmoid function approximation (used to approximate the indicator function) method proposed by [15], it is rather complex and its performance is much worse than the suboptimal scheme we will provide in subsection $B$.

Let $\mathcal{P}=\left\{p_{1}, \ldots, p_{L}\right\}$, where $p_{1}>\cdots>p_{L} \geq 0$, and the corresponding channel partitioning $\mathcal{R}_{1}, \ldots, \mathcal{R}_{L}$ denote an optimal solution to Problem (5). Let $p\left(\mathcal{I}\left(g_{0}, g_{1}\right)\right)$ represent the mapping from instantaneous $\left(g_{0}, g_{1}\right)$ information to the allocated power level. We can obtain the following result:

Lemma 1: Let $\left\{v_{1}, \ldots, v_{L}\right\}$ denote the optimum quantization thresholds on the $g_{1}$ axis $\left(0<v_{1}<\cdots<v_{L}\right)$ and $\left\{s_{1}, \ldots, s_{L-1}\right\}$ indicate the optimum quantization thresholds on the $g_{0}$ axis $\left(0<s_{1}<\cdots<s_{L-1}\right)$. Then we have $\forall j, j=1, \ldots, L-1$ :

$p\left(\mathcal{I}\left(g_{0}, g_{1}\right)\right)=\left\{\begin{array}{lc}p_{j}, & v_{j} \leq g_{1}<v_{j+1}, 0 \leq g_{0}<s_{j} \\ p_{L}, & \text { otherwise }\end{array}\right.$ 
where $v_{j}=\frac{c}{p_{j}}, j=1, \ldots, L$, and for $\forall j, j=1, \ldots, L-1$, when $\mu>0, s_{j}=\frac{1}{\mu\left(p_{j}-p_{L}\right)}-\frac{\lambda}{\mu}$, while when $\mu=0, s_{j}=$ $\infty$, then condition $0 \leq g_{0}<s_{j}$ boils down to $\lambda<\frac{1}{p_{j}-p_{L}}$. The region $\mathcal{R}_{L}$ includes two parts : the set $\mathcal{R}_{L 1}=\left\{\left(g_{0}, g_{1}\right)\right.$ : $\left.v_{j} \leq g_{1}<v_{j+1}, g_{0} \geq s_{j}, \forall j=0, \ldots, L-1\right\}$ with $s_{0}=$ $0, v_{0}=0$ and the set $\mathcal{R}_{L 2}=\left\{\left(g_{0}, g_{1}\right): g_{1} \geq v_{L}, g_{0} \geq 0\right\}$. The entire set $\mathcal{R}_{L 1}$ is in outage.

Remark 2: When $\mu>0$, which implies that the AIP constraint is active, from Lemma 1, the optimum partition regions possess a stepwise structure, as shown in Fig.2. When $\mu=0$, i.e, the AIP constraint is inactive and only ATP constraint is active (we must have $\lambda>0$ ), Problem (4) becomes a scalar quantization problem involving quantizing $g_{1}$ only, and Lemma 1 reduces to :

$p\left(\mathcal{I}\left(g_{1}\right)\right)=\left\{\begin{array}{l}p_{j}, \quad v_{j} \leq g_{1}<v_{j+1}, \forall j, j=1, \ldots, L-1 \\ p_{L}, \quad \text { otherwise }\end{array}\right.$

where $\lambda<\frac{1}{p_{j}-p_{L}}, \forall j=1,2, \ldots, L-1$ and the two subregions of $\mathcal{R}_{L}$ become $\mathcal{R}_{L 1}=\left\{g_{1}: 0 \leq g_{1}<v_{1}\right\}$ and $\mathcal{R}_{L 2}=\left\{g_{1}: g_{1} \geq v_{L}\right\}$, and $\mathcal{R}_{L 1}$ is in outage.

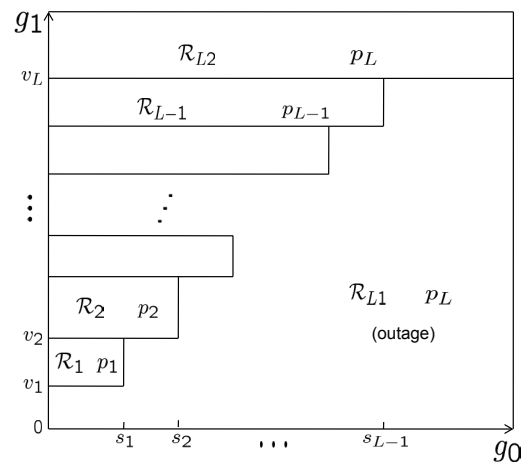

Fig. 2. The 'stepwise structure' of optimum quantization regions for $\mu>0$ case

From Lemma 1, (due to the fading channels being independently exponentially distributed with unity mean) Problem (4) becomes,

$$
\begin{aligned}
& \min _{p_{j} \geq 0, \forall j} P_{\text {out }}^{L}=1-e^{-v_{1}}+\sum_{j=1}^{L-1}\left(e^{-v_{j}}-e^{-v_{j+1}}\right) e^{-s_{j}} \\
& \text { s.t. } p_{L}+\sum_{j=1}^{L-1}\left(p_{j}-p_{L}\right)\left(e^{-v_{j}}-e^{-v_{j+1}}\right)\left(1-e^{-s_{j}}\right) \leq P_{a v} \\
& p_{L}+\sum_{j=1}^{L-1}\left(p_{j}-p_{L}\right) \\
& \quad \times\left(e^{-v_{j}}-e^{-v_{j+1}}\right)\left(1-e^{-s_{j}}\left(1+s_{j}\right)\right) \leq Q_{a v}
\end{aligned}
$$

where $P_{\text {out }}^{L}$ denotes the outage probability with $B=\log _{2} L$ bits feedback QPA, $v_{j}=\frac{c}{p_{j}}, j=1, \ldots, L$ and for $\forall j, j=$ $1, \ldots, L-1$, when $\mu>0, s_{j}=\frac{1}{\mu\left(p_{j}-p_{L}\right)}-\frac{\lambda}{\mu}$, whereas when $\mu=0, s_{j}=\infty$. Although the above optimization problem may be verified to be non-convex, we can employ the KKT necessary conditions to find a local minimum for Problem (8). Taking the partial derivative of first order of the Lagrangian of Problem (8) over $p_{j}, j=1, \ldots, L-1$, and setting it to zero, we can obtain,

$$
p_{j+1}=\frac{c}{v_{j}-\ln \left(1-\frac{\frac{c}{p_{j}^{2}}\left[\hat{f}\left(p_{j-1}\right)-\hat{f}\left(p_{j}\right)\right]}{\lambda\left(1-e^{-s_{j}}\right)+\mu\left(1-e^{-s_{j}}\left(1+s_{j}\right)\right)}\right)},
$$

where $\hat{f}\left(p_{0}\right)=1$ and $\hat{f}\left(p_{j}\right)=\left(p_{j}-p_{L}\right)(\lambda+\mu(1-$ $\left.\left.e^{-s_{j}}\right)\right), 1 \leq j \leq L-1$. Equating the partial derivative of the Lagrangian function of Problem (8) over $p_{L}$ to zero gives,

$$
\begin{aligned}
& \sum_{j=1}^{L-1}\left(e^{-v_{j}}-e^{-v_{j+1}}\right)\left[\lambda\left(1-e^{-s_{j}}\right)+\mu\left(1-e^{-s_{j}}\left(1+s_{j}\right)\right)\right] \\
& +e^{-v_{L}} \frac{c}{p_{L}^{2}} \hat{f}\left(p_{L-1}\right)=\lambda+\mu
\end{aligned}
$$

Thus, for fixed values $\lambda$ and $\mu$, we need to solve the $L$ equations given by (9), (10) to obtain the power codebook.

(7) Given $p_{1}$ and $p_{L}$, from (9) we can successively compute $p_{2}, \ldots, p_{L-1}$, and then we can jointly solve the equation (9) with $j=L-1$ and equation (10) numerically for $p_{1}$ and $p_{L}$. The optimal value of $\lambda$ and $\mu$ can be obtained by a subgradient method, i,e. by updating $\lambda$ and $\mu$ until convergence using Step 2. One can thus repeat the above two steps (i.e, given $\lambda$ and $\mu$ find the optimal power levels, and then using the resulting optimal power levels update $\lambda$, $\mu$ ) iteratively until a satisfactory convergence criterion is met.

\section{B. Suboptimal QPA Algorithm}

When the number of feedback bits $B$ (or alternatively, $L$ ) goes to infinity, we can obtain the following Lemma that allows us to obtain a suboptimal but computationally efficient quantized power allocation algorithm for large but finite $L$.

Lemma 2: $\lim _{L \rightarrow \infty} p_{L}=0$

Remark 3: Lemma 2 shows that regardless of whether $\mu>0$ or $\mu=0$, with high rate quantization, the power level for the last region $\mathcal{R}_{\mathcal{L}}$ approaches zero, which also implies the following as $L \rightarrow \infty$ :

1) The non-outage part of $\mathcal{R}_{\mathcal{L}}$, given by $\mathcal{R}_{\mathcal{L} 2}$, disappears gradually. In other words, $\mathcal{R}_{\mathcal{L}} \rightarrow \mathcal{R}_{\mathcal{L}_{1}}$. Thus, when $L \rightarrow \infty$, $\mathcal{R}_{\mathcal{L}}$ becomes the outage region assigned with zero power.

2) When $\mu>0$, the quantization thresholds on $g_{0}$ axis $s_{j} \rightarrow s_{j}^{\prime}$ (where $s_{j}^{\prime}=\frac{1}{\mu p_{j}}-\frac{\lambda}{\mu}$ ), which gives $v_{j}=c \lambda+c \mu s_{j}^{\prime}$, and it means all the points given by coordinates $\left(s_{j}^{\prime}, v_{j}\right)$ lie on the line of $g_{1}=c \lambda+c \mu g_{0}$. Therefore, as $L \rightarrow \infty$, the stepwise shape of the structure in $\mu>0$ case (i.e, the boundary between non-outage and outage regions) approaches the straight line $g_{1}=c \lambda+c \mu g_{0}$, which is consistent with the full CSI-based power allocation result in (2).

Thus, when $L$ is large, applying Lemma 2 (i.e, $p_{L} \rightarrow 0$ ) to Problem (8), the above $L$ KKT conditions (9) and (10) can be simplified into $L-1$ equations:

$$
\begin{aligned}
& p_{j+1}=\frac{c}{v_{j}-\ln \left(1-\frac{\frac{c}{p_{j}^{2}}\left[p_{j-1}\left(\lambda+\mu\left(1-e^{-s_{j-1}^{\prime}}\right)\right)-p_{j}\left(\lambda+\mu\left(1-e^{-s_{j}^{\prime}}\right)\right)\right]}{\lambda\left(1-e^{-s_{j}^{\prime}}\right)+\mu\left(1-e^{-s_{j}^{\prime}}\left(1+s_{j}^{\prime}\right)\right)}\right)} \\
& j=1, \ldots, L-2 \text {; } \\
& \lambda\left(1-e^{-s_{L-1}^{\prime}}\right)+\mu\left(1-e^{-s_{L-1}^{\prime}}\left(1+s_{L-1}^{\prime}\right)\right)=\frac{c}{p_{L-1}^{2}}\left[p_{L-2}\right. \\
& \left.\left(\lambda+\mu\left(1-e^{-s_{L-2}^{\prime}}\right)\right)-p_{L-1}\left(\lambda+\mu\left(1-e^{-s_{L-1}^{\prime}}\right)\right)\right]
\end{aligned}
$$

where when $\mu>0$, the quantization thresholds on the $g_{0}$ axis are given by $s_{j}^{\prime}=\frac{1}{\mu p_{j}}-\frac{\lambda}{\mu}, s_{0}^{\prime}=0$, and $p_{0}=\frac{1}{\lambda+\mu s_{0}^{\prime}}$, 
while when $\mu=0, s_{j}^{\prime}=\infty, s_{0}^{\prime}=0$, and $p_{0}=\frac{1}{\lambda}$. Thus, for given values of $\lambda$ and $\mu$, starting with a specific value of $p_{1}$, we can successively compute $p_{2}, \ldots, p_{L-1}$ using the first equation of (11) (recall that $v_{j}=\frac{c}{p_{j}}$ ). Then the second equation in (11) becomes an equation in $p_{1}$ only, which can be solved easily using a suitable nonlinear equation solver. We call this suboptimal QPA algorithm as 'Zero Power in Outage Region Approximation'(ZPiORA).

\section{ASYMPTOTIC OUTAGE BEHAVIOUR OF QPA UNDER HIGH RESOLUTION QUANTIZATION}

In this section, we derive a number of asymptotic expressions for the SU outage probability when the number of feedback bits approaches infinity. To this end, we first derive some useful properties regarding the quantizer structure at high rate quantization:

Lemma 3: As the number of quantization regions $L \rightarrow \infty$, we can obtain the following results:

1) when $\mu>0$, the optimum quantization thresholds on the $g_{0}$ axis satisfy

$$
s_{1}^{\prime}-s_{0}^{\prime} \approx s_{2}^{\prime}-s_{1}^{\prime} \approx \cdots \approx s_{L-1}^{\prime}-s_{L-2}^{\prime}
$$

where $s_{j}^{\prime}=\frac{1}{\mu p_{j}}-\frac{\lambda}{\mu}, j=1, \ldots, L-1$ and $s_{0}^{\prime}=0$.

2) when $\mu=0$, the optimum quantization thresholds on the $g_{1}$ axis satisfy

$$
\frac{v_{1}}{v_{0}} \approx \frac{v_{2}}{v_{1}} \cdots \approx \frac{v_{L-1}}{v_{L-2}}
$$

where $v_{j}=\frac{c}{p_{j}}, j=1, \ldots, L-1$ and here $v_{0}=c \lambda$.

Lemma 4: In the high rate quantization regime, as $L \rightarrow$ $\infty$, we have

$$
\begin{aligned}
& \sum_{j=1}^{L-1}\left(e^{-v_{j}}-e^{-v_{j+1}}\right)\left[\lambda\left(1-e^{-s_{j}^{\prime}}\right)+\mu\left(1-e^{-s_{j}^{\prime}}\left(1+s_{j}^{\prime}\right)\right)\right] \\
& \approx \frac{\lambda P_{a v}+\mu Q_{a v}}{L-1} \sum_{j=1}^{L-1} \frac{1}{p_{j}} .
\end{aligned}
$$

where when $\mu>0, s_{j}^{\prime}=\frac{1}{\mu p_{j}}-\frac{\lambda}{\mu}$, whereas when $\mu=$ $0, s_{j}^{\prime}=\infty$, and (14) simplifies to $c e^{-v_{1}} \approx \frac{P_{a v}}{L-1} \sum_{j=1}^{L-1} v_{j}$ with $v_{j}=\frac{c}{p_{j}}$.

With Lemma 3 and Lemma 4, the main result of this section can be obtained in the Theorem below.

Theorem 1: The asymptotic SU outage probability for a large number of feedback bits can be approximately expressed as,

1) when $\mu>0$,

$$
P_{\text {out }}^{L} \approx 1-e^{-c \lambda_{f}^{*}}\left[1-\left(1-e^{-\frac{a}{L}}\right) \frac{1-e^{-a\left(1+\frac{1}{c \mu_{f}^{*}}\right)}}{1-e^{-\frac{a\left(1+\frac{1}{\left.c \mu_{f}^{*}\right)}\right.}{L}}}\right],
$$

where $a$ is a constant satisfying the equation: $\left(\lambda_{f}^{*} P_{a v}+\right.$ $\left.\mu_{f}^{*} Q_{a v}\right)\left(\lambda_{f}^{*}+\frac{a}{2 c}\right) e^{c \lambda_{f}^{*}} \approx\left[\left(\lambda_{f}^{*}+\mu_{f}^{*}\right)\left(1-\frac{c \mu_{f}^{*}}{1+c \mu_{f}^{*}}(1-\right.\right.$ $\left.\left.\left.e^{-a\left(1+\frac{1}{c \mu_{f}^{*}}\right)}\right)\right)-\frac{c\left(\mu_{f}^{*}\right)^{2}}{\left(1+c \mu_{f}^{*}\right)^{2}}\left(1-e^{-a\left(1+\frac{1}{c \mu_{f}^{*}}\right)}\left(1+a\left(1+\frac{1}{c \mu_{f}^{*}}\right)\right)\right)\right]$. We also have $\lim _{L \rightarrow \infty} P_{\text {out }}^{L}=1-e^{-c \lambda_{f}^{*}}\left[1-\frac{1-e^{-a\left(1+\frac{1}{c \mu_{f}^{*}}\right)}}{1+\frac{1}{c \mu_{f}^{*}}}\right]$. 2) when $\mu=0, P_{\text {out }}^{L} \approx 1-e^{-c \lambda_{f}^{*}\left(1+\frac{\beta}{L}\right)}$, where $\beta$ is a constant given by $e^{-c \lambda_{f}^{*}} \approx \lambda_{f}^{*} P_{a v} \frac{e^{\beta}-1}{\beta}$. In this case we also have $\lim _{L \rightarrow \infty} P_{\text {out }}^{L}=1-e^{-c \lambda_{f}^{*}}$.

\section{Numerical Results}

In this section, we will examine the outage probability performance of the $\mathrm{SU}$ in a narrowband spectrum sharing system with the proposed power allocation strategies via numerical simulations. The required transmission rate is taken to be $r_{0}=0.25$ nats per channel use.

Fig. 3 displays the SU outage probability performance of the suboptimal algorithm ZPiORA versus $P_{a v}$ with feedback bits $B=\{1,2\}$, under $Q_{a v}=-5 \mathrm{~dB}$ and $Q_{a v}=0 \mathrm{~dB}$ respectively, and compares these results with the corresponding outage performance of the optimal QPA. As observed from Fig. 3, when $Q_{a v}=-5 \mathrm{~dB}$, with $B$ fixed, the outage performances of ZPiORA and corresponding optimal QPA almost overlap with each other. When $Q_{a v}=0 \mathrm{~dB}$ and $P_{a v} \leq-5 \mathrm{~dB}$, with the same number of feedback bits, the outage performances of these two methods are still indistinguishable; and with $P_{a v}>-5 \mathrm{~dB}$, the outage performance gap between ZPiORA and corresponding optimal QPA is decreasing with increasing B. For example, with 1 bit feedback, at $P_{a v}=10 \mathrm{~dB}$, the outage gap between ZPiORA and optimal QPA is 0.0347 , but with 2 bits of feedback, the outage performance of these two methods are very close to each other, which agrees with Lemma 2 that ZPiORA is a near-optimal algorithm for large number of feedback bits.

Fig. 4 illustrates the outage performance of SU with

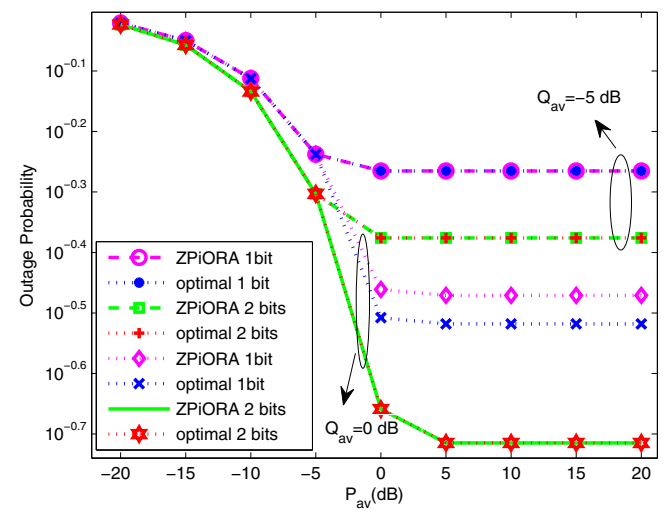

Fig. 3. Outage probability performance comparison between ZPiORA and optimal QPA

optimal QPA strategy versus $P_{a v}$ with feedback bits $B=$ $\{2,4,6\}$, under $Q_{a v}=-5 \mathrm{~dB}$ and $Q_{a v}=0 \mathrm{~dB}$ respectively, and studies the effect of increasing the number of feedback bits on the outage performance. For comparison, we also plot the corresponding SU outage performance with full CSI case. Since ZPiORA is an efficient suboptimal method for large number of feedback bits, we employ ZPiORA to obtain the outage performance instead of using optimal QPA for $B=6$ bits. First, it can be easily observed that all the outage curves decrease gradually as $P_{a v}$ increases until $P_{a v}$ reaches a certain threshold, when the outage probability attains a floor. This is due to the fact that in the high $P_{a v}$ regime, the AIP constraint dominates. For a fixed number of feedback bits, the higher $Q_{a v}$ is, the smaller the resultant outage probability is, since higher $Q_{a v}$ means PU can tolerate more interference. Fig. 4 also illustrates that for fixed $Q_{a v}$, introducing one extra bit of feedback substantially reduces the outage gap between QPA and the perfect CSI case. To be specific, for $Q_{a v}=0 \mathrm{~dB}$ 
and $P_{a v}=10 \mathrm{~dB}$, with 2 bits, 4 bits and 6 bits of feedback, the outage gaps with full CSI case are approximately 0.1083 , 0.0249 and 0.006979 respectively. And for any $Q_{a v}$, only 6 bits of feedback seem to result in an SU outage performance very close to that with full CSI case.

Figure 5 depicts the asymptotic outage probability be-

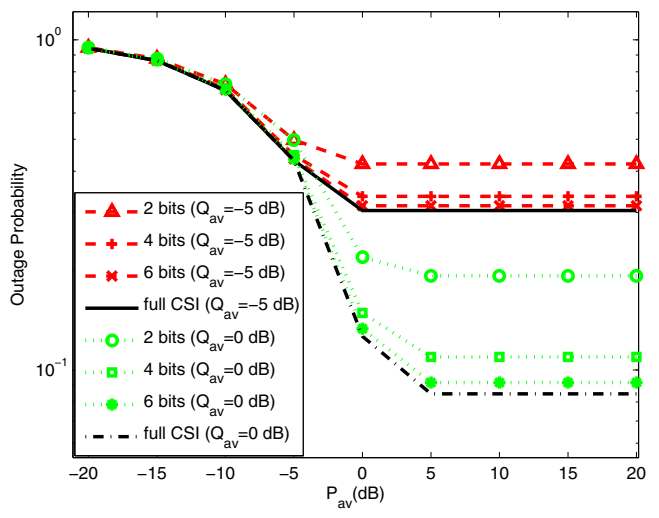

Fig. 4. Effect of increasing feedback bits on outage performance of SU

havior of SU versus the number of quantization level $L$ at $Q_{a v}=0 \mathrm{~dB}, P_{a v}=10 \mathrm{~dB}$, and compares the result with the corresponding full CSI performance. It can be seen from Figure 5 that the outage decreases as the number of quantization level $L$ increases, however, as $L$ increases beyond a certain number ( $L \geq 2^{8}$, i.e, $B \geq 8$ bits), the outage probability curve starts to saturate and approaches the full CSI performance. This further confirms that only a small number of feedback bits is enough to obtain an outage performance close to the perfect CSI-based performance.

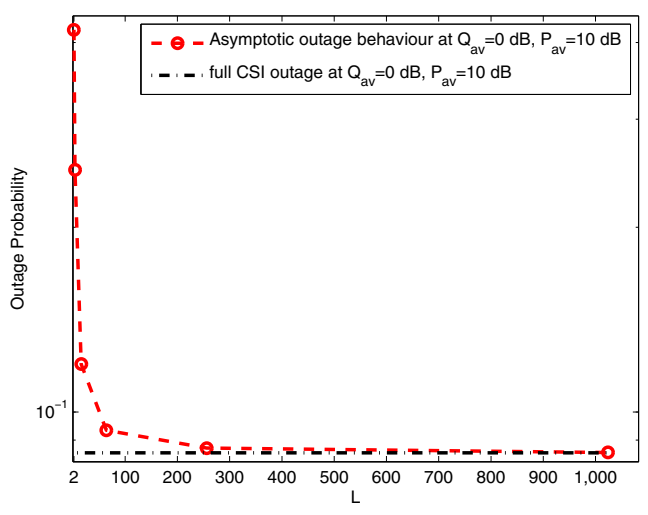

Fig. 5. Asymptotic outage behavior versus the number of quantization level L

\section{CONCLUSIONS}

In this paper, we designed locally optimal power allocation algorithms for secondary outage probability minimization with quantized CSI information for a narrowband spectrum sharing cognitive radio framework under an ATP constraint at SU-TX and an AIP constraint at PU-RX. We proved that the optimal channel partition structure has a "stepwise" pattern based on which an efficient optimal power codebook design algorithm is provided. In the case of a large number of feedback bits, we proposed a novel low-complexity suboptimal algorithm ZPiORA. We also derived approximate expressions for the asymptotic behavior of the SU outage probability for a large number of feedback bits, which are shown to be extremely accurate. Although the presented power codebook design methods result in locally optimal solutions (due to the non-convexity of the quantized power allocation problem), numerical results illustrate that only 6 bits of feedback result in SU outage performance very close to that obtained with full CSI at the SU transmitter.

\section{REFERENCES}

[1] J.M. Peha, "Sharing Spectrum Through Spectrum Policy Reform and Cognitive Radio," Proc. of the IEEE, vol. 97, no. 4, pp. 708-719, Apr. 2009.

[2] J. Mitola III, "Cognitive radio for flexible mobile multimedia communications," IEEE Int. Workshop on Mobile Multimedia Commun. $($ МоМиС) , San Diego, CA, USA, Nov. 1999, pp. 3-10.

[3] A. Goldsmith, S.A. Jafar, I. Maric, and S. Srinivasa, "Breaking spectrum gridlock with cognitive radios: an information theoretic perspective," Proceedings of the IEEE, vol. 97, no. 5, pp. 894-914, May 2009.

[4] X. Kang, Y. Liang, A. Nallanathan, H.K. Garg and R. Zhang, "Optimal power allocation for fading channels in cognitive radio networks: Ergodic capacity and outage capacity," IEEE Trans. Wireless Commun., vol. 8, no. 2, pp. 940-950, Feb. 2009.

[5] A. Ghasemi and E. S. Sousa, "Fundamental limits of spectrum-sharing in fading environments," IEEE Trans. Wireless Commun., vol. 6, no. 2, pp. 649-658, Feb. 2007.

[6] L. Musavian and S. Aissa, "Capacity and power allocation for spectrumsharing communications in fading channels," IEEE Trans. Wireless Commun., vol. 8, no. 1, pp. 148-156, Jan. 2009.

[7] L. Musavian and S. Aissa, "Fundamental capacity limits of cognitive radio in fading environments with imperfect channel information," IEEE Trans. Commun., vol. 57, no. 11, pp. 3472-3480, Nov. 2009.

[8] H.A. Suraweera, P.J. Smith and M. Shafi, "Capacity Limits and Performance Analysis of Cognitive Radio With Imperfect Channel Knowledge," IEEE Transactions on Vehicular Technology, vol. 59, no. 4, pp. 1811-1822, February 2010.

[9] K. Huang and R. Zhang "Cooperative feedback for multi-antenna cognitive radio networks ," IEEE Transactions on Signal Processing, vol. 59, no. 2, pp. 747-758, February 2011.

[10] L. Zhang, Y-C. Liang, Y. Xin and H.V. Poor, "Robust Cognitive Beamforming with Partial Channel State Information," IEEE Transactions on Wireless Communications, vol. 8, no. 8, pp. 4143-4153, August 2009.

[11] A.G. Marques, X. Wang and G.B. Giannakis, "Dynamic Resource Management for Cognitive Radios Using Limited-Rate Feedback," IEEE Transactions on Signal Processing, vol. 57, no. 9, pp. 3651-3666, September 2009.

[12] Y. Y. He and S. Dey "Power allocation in spectrum sharing cognitive radio networks with quantized channel information," IEEE Trans. Commun., vol. 59, no. 6, pp. 1644-1656, June 2011,

[13] R. Zhang, "On peak versus average interference power constraints for protecting primary users in cognitive radio networks," IEEE Trans. Wireless Commun., vol. 8, no. 4, pp. 2112-2120, April 2009.

[14] J. C. Spall, "Implementation of the simultaneous perturbation algorithm for stochastic optimization," IEEE Transactions on Aerospace and Electronic Systems, vol. 34, no. 3, pp. 817-823, Jul. 1998.

[15] B. Khoshnevis and Wei Yu,"Joint Power Control and Beamforming Codebook Design for MISO Channels with Limited Feedback," IEEE Global Telecommunications Conference(GLOBECOM 2009), Honolulu, HI, USA, Dec. 2009, pp. 1 - 6.

[16] Y.Y. He and S. Dey, "Power Allocation for Outage Minimization in Cognitive Radio Networks with Limited Feedback," available at http://arxiv.org/abs/1111.2640. 